\title{
AN ELEMENTARY DERIVATION OF PONTRYAGIN'S MAXIMUM PRINCIPLE OF OPTIMAL CONTROL THEORY
}

\author{
J. M. BLATT and J. D. GRAYY
}

(Received 14 January 1976)

(Revised 1 April 1977)

\begin{abstract}
Pontryagin's maximum principle is derived by elementary mathematical techniques. The conditions on the functions which enter are generally somewhat more stringent than in Pontryagin's derivation, but one (practically very awkward) condition of Pontryagin can be relaxed: continuity in the time variable can be replaced by a much weaker condition.
\end{abstract}

\section{Introduction}

In Pontryagin's work [8], his maximum principle is derived using the apparatus of Lebesgue theory of functions of real variables. Ever since, it has been generally accepted that such techniques are essential for modern control theory. Some possible exceptions to this may be found in Halkin [6], Bryant and Mayne [3], and Halkin and Neustadt [7], where the principle is derived through a non-linear programming problem in an infinite dimensional space. Regardless, when even simpler derivations are presented in textbooks [5] they are generally labelled "heuristic" and the reader is referred back to Pontryagin for a rigorous derivation.

In this paper, we give a rigorous derivation of one form of Pontryagin's maximum principle by use of elementary techniques only (for example, the mean value theorem, Taylor's theorem with remainder term, the Picard existence theorem for differential equations). We do not use Lebesgue theory at all. Our derivation is essentially the same as the "heuristic derivation" of Hadley and Kemp [5] but enough details are filled in to show that it can be made quite rigorous.

It is necessary, of course, to put conditions on the functions more stringent than those postulated by Pontryagin. However, our conditions are satisfied in most practical cases. The use of elementary techniques gives a much better intuitive 
understanding of what is really going on. And, last but not least, one of Pontryagin's conditions (continuity of the functions $f(x, v, t)$ and $f_{0}(x, v, t)$ as functions of $t$ ) can be relaxed considerably: we can replace continuity by piecewise continuity. In practice, this is a very desirable relaxation of conditions.

The purpose of this paper is didactic, to show how one can gain insight. For this reason, we concentrate on the simplest control problem which exhibits the essential features of interest to us. The only serious simplification is (2) below. The simplifications we adopt are:

(1) There is a fixed planning horizon $T$, that is, the quantity to be minimized is

$$
x_{0} \equiv \int_{0}^{T} f_{0}[x(t), v(t), t] d t,
$$

where $T>0$ is given once and for all.

(2) The endpoint is "free", that is, there is no condition on the final value $x(T)$ of the state variable.

(3) There is only one degree of freedom, that is, the state variable $x(t)$ is a real-valued function of $t$, not a vector-valued function of $t$. However, much of what follows can readily be extended to the vector-valued case.

(4) The control variable $v(t)$ takes only real values (that is, it is not a vector with more than one component), and these values lie within a control set $\Omega$ which is some subset of the real numbers. We permit $\Omega$ to be a finite discrete set or a finite set of non-overlapping closed intervals of type $u_{i} \leqslant v \leqslant w_{i}$, or any combination of these two; but nothing more complicated. Furthermore, the admissible controls $v(t)$ are piece-wise continuous functions of $t$, with at most a finite number of points of discontinuity in the interval $0 \leqslant t \leqslant T$. We take $v(t)$ to be continuous to the left at a point of discontinuity, that is, $\lim _{e \rightarrow 0+} v(t-\varepsilon)=v(t)$ for all $t$.

(5) The time-development of $x(t)$ is governed by

$$
\frac{d x}{d t}=f(x, v, t)
$$

together with the initial condition

$$
x(0)=b,
$$

where $b$ is a given constant.

(6) We now impose conditions on $f_{0}(x, v, t)$ and $f(x, v, t)$, designed to ensure the existence and good behaviour of $x(t)$ and $x_{0}(t)$ for all $0 \leqslant t \leqslant T$ and all admissible controls. Our conditions (which are more stringent than those of Pontryagin [1]) enable us to prove existence of our solutions (though not of an optimal solution). We shall assume the following: There exist numbers $\alpha, \beta, \gamma, \delta$, satisfying

$$
\alpha<\beta \leqslant b \leqslant \gamma<\delta,
$$


such that $f(x, v, t)$ and $f_{0}(x, v, t)$ are real-valued functions defined on the region

$$
R: \alpha \leqslant x \leqslant \delta, \quad v \in \Omega, \quad 0 \leqslant t \leqslant T .
$$

We note that the region $R$ is closed and compact. The functions $f$ and $f_{0}$ are assumed to be twice differentiable with respect to $x$ everywhere in $R$, with bounded second derivatives

$$
\left|\frac{\partial^{2} f}{\partial x^{2}}\right| \leqslant A,\left|\frac{\partial^{2} f_{0}}{\partial x^{2}}\right| \leqslant A \quad \text { on } R .
$$

Furthermore, the first derivatives and the functions themselves are bounded:

$$
\begin{gathered}
\left|\frac{\partial f}{\partial x}\right| \leqslant B, \quad\left|\frac{\partial f_{0}}{\partial x}\right| \leqslant B \quad \text { on } R, \\
|f| \leqslant M, \quad\left|f_{0}\right| \leqslant M \quad \text { on } R .
\end{gathered}
$$

The functions $f(\cdot, v, \cdot)$ and $f_{0}(\cdot, v, \cdot)$ are continuous; and the functions $f(\cdot, \cdot, t)$ and $f_{0}(\cdot, \cdot, t)$ are piecewise continuous with at most a finite number of points of discontinuity in $[0, T]$. At points of discontinuity $f(\cdot, \cdot, t)$ and $f_{0}(\cdot, \cdot, t)$ are left-hand continuous:

$$
\begin{aligned}
& f(\cdot, v, \cdot) \text { and } f_{0}(\cdot, v, \cdot) \text { are continuous on } \Omega, \\
& f(\cdot, \cdot, t) \text { and } f_{0}(\cdot, \cdot, t) \text { are piecewise continuous on }[0, T]
\end{aligned}
$$

\section{Existence and properties of trajectories}

In this section, we prove the existence and uniqueness of the solution $x(t)$ of (1.2) and (1.3) for every admissible control $v(t)$.

First we impose two further restrictions on $f$ (but not on $f_{0}$ ), with the intention of ensuring that $x(t)$ remains bounded: $\beta \leqslant x(t) \leqslant \gamma$, for all $t$ in $0 \leqslant t \leqslant T$. These restrictions are:

$$
\begin{aligned}
& f(x, v, t)>0 \text { for } \alpha \leqslant x \leqslant \beta, \quad v \in \Omega, \quad 0 \leqslant t \leqslant T, \\
& f(x, v, t)<0 \text { for } \gamma \leqslant x \leqslant \delta, \quad v \in \Omega, \quad 0 \leqslant t \leqslant T .
\end{aligned}
$$

Intuitively, (2.1) and (2.2) ensure that $x(t)$ can never "penetrate" the region $\alpha \leqslant x \leqslant \beta$. At time $t=0, x(0)=b$ satisfies $\beta \leqslant x(0) \leqslant \gamma$, by (1.4). As soon as $x(t)$ falls down to $\beta$ or below it, the differential equation (1.2) implies a positive derivative $d x / d t$ which forces $x$ to move upwards with increasing $t$. Similarly, should $x(t) \geqslant \gamma$ for some $t$, the combination of (1.2) and (2.2) implies $d x / d t<0$, so $x$ is forced downwards again, back into the region

$$
\beta \leqslant x(t) \leqslant \gamma \text { all } t \text { in } 0 \leqslant t \leqslant T .
$$


This heuristic discussion will be replaced by a proper derivation subsequently; but it should serve to motivate the conditions (2.1) and (2.2). An alternative condition known to guarantee that $x(t)$ does not excape is $|f(x, v, t)| \leqslant M(1+|x|)$ for some constant $M$ [3]. (This condition is meaningful in the vector-valued case too.)

Such conditions are often, indeed usually, satisfied in practical situations. The uncontrolled system is often naturally stable, that is, $x(t)$ remains within some "region of stability" $(\beta \leqslant x \leqslant \gamma)$ in any case, no matter what control $v(t)$ we impose. There is no suggestion that $\beta \leqslant x \leqslant \gamma$ is a narrow region of the $x$-axis, merely that $x(t)$ cannot go off to infinity. Of course, there are some occasions when we are faced with the prospect of having to control a naturally unstable system; but such control is much more difficult to achieve.

If one is prepared to assume existence of a solution [8] then this section can be skipped. Also, in that case conditions (2.1) and (2.2) need not be imposed.

We start from the identity

$$
f\left(x^{\prime}, v, t\right)-f\left(x^{\prime \prime}, v, t\right)=\int_{x^{\prime \prime}}^{x \prime} \frac{\partial f}{\partial x} d x
$$

We take the absolute value on both sides, and use (1.7a) to obtain the bound

$$
\left|f\left(x^{\prime}, v, t\right)-f\left(x^{\prime \prime}, v, t\right)\right| \leqslant B\left|x^{\prime}-x^{\prime \prime}\right| \text { on } R .
$$

Now let $v(t)$ be any admissible control, that is, for each $t, v(t) \in \Omega$, and $v(t)$ is a piecewise continuous function of $t$ with at most finitely many points of discontinuity within $[0, T]$. Define

$$
g(x, t)=f[x, v(t), t], \quad \alpha \leqslant x \leqslant \delta, \quad 0 \leqslant t \leqslant T .
$$

Since $f$ is twice differentiable with respect to $x$, continuous in $v$, and piecewise continuous in $t, g(x, t)$ is twice differentiable in $x$ and piecewise continuous in $t$. The points of discontinuity, if any, of $g(\cdot, t)$ may arise from two sources: (i) discontinuities in $f(\cdot, \cdot, t)$; or (ii) discontinuities in $v(t)$. But there are at most a finite number of such values of $t$, from either cause.

Clearly $(1.7 b)$ implies

$$
|g(x, t)| \leqslant M \text { on } S \text {, }
$$

where $S$ is the region

$$
S: \alpha \leqslant x \leqslant \delta, \quad 0 \leqslant t \leqslant T .
$$

Furthermore, (2.5) implies a Lipschitz condition:

$$
\left|g(x, t)-g\left(x^{\prime}, t\right)\right| \leqslant B\left|x-x^{\prime}\right| \text { on } S,
$$

where the constant $B$ is the one which appears in (1.7a), and is independent of $x, x^{\prime}$ and $t$. 
We shall make use of the Picard existence proof of solutions of

$$
\frac{d x}{d t}=g(x, t)
$$

with initial condition

$$
x(0)=b,
$$

in the form in which this proof is given in Agnew's book [1]. In this proof, an important role is assigned to the triangular region $\tilde{T}$ :

$$
\tilde{T}: t_{0} \leqslant t \leqslant t_{1}, \quad|x-b| \leqslant M|t| .
$$

The point $x=b, t=0$ must be within $\widetilde{T}$, and the Lipschitz condition (2.9) must be satisfied everywhere within $\tilde{T}$.

Since we are interested in the forward solution (positive $t$ ) only, we put $t_{0}=0$, and $t_{1}=\Delta t$, where $\Delta t$ is defined by

$$
\Delta t=\min \left(\frac{\beta-\alpha}{M}, \frac{\delta-\gamma}{M}\right) .
$$

By (1.4), we know that $\Delta t$ is not zero; it also follows from (1.4) that the region $\tilde{T}$, (2.12), with $t_{0}=0$ and $t_{1}=\Delta t$, lies entirely inside the region $S$, (2.8). Hence (2.9) ensures that the Lipschitz condition is satisfied in the form postulated by Agnew [1].

The one point where we fail to meet Angew's conditions is continuity: $g(x, t)$, (2.6), is only piecewise continuous in $t$, not continuous.

However, $g(x, t)$ is bounded in $R$ and (being piecewise continuous) is integrable in the Riemann sense. This implies that Agnew's lemma 15.81 (p. 312) goes through with only one, obvious, modification: if $g(x, t)$ has a discontinuity at $t=\theta$, say, then the integral formulation of (2.10) and (2.11),

$$
x(t)=b+\int_{0}^{t} g\left[x\left(t^{\prime}\right), t^{\prime}\right] d t^{\prime}
$$

may not be equivalent to (2.10) at $t=\theta$. The same remark applies to the iteration scheme based on (2.14)

$$
x_{n+1}(t)=b+\int_{0}^{t} g\left[x_{n}\left(t^{\prime}\right), t^{\prime}\right] d t^{\prime} .
$$

With this proviso, the entire proof in Agnew [1] goes through. We do not reproduce this proof here, but we remark that the proof is thoroughly elementary, establishing explicit bounds to prove the uniform and absolute convergence of the sequence $x_{n}(t)$ to a unique function $x(t)$ which satisfies (2.14) and hence satisfies (2.10) and (2.11) everywhere except at a finite set of points $t$ for which $g(x, t)$ is discontinuous.

All this is restricted to the interval $[0, \Delta t]$, where $\Delta t$ is given by (2.13). We now proceed to extend the solution so obtained to the entire planning period $[0, T]$ using 
the following notation:

$$
\begin{aligned}
& t_{n} \equiv n \Delta t, \quad x_{n} \equiv x\left(t_{n}\right), \quad n=0,1,2, \ldots, \\
& \Delta S_{n}: t_{n-1} \leqslant t \leqslant t_{n}, \quad\left|x-x_{n-1}\right| \leqslant M\left|t-t_{n-1}\right| .
\end{aligned}
$$

LEMma. Let $x(t)$ be defined on $t_{n-1} \leqslant t \leqslant t_{n}$ and satisfy the differential equation (2.10) with initial condition $x\left(t_{n-1}\right)=x_{n-1}$ everywhere in $\left[t_{n-1}, t_{n}\right]$ except at points of discontinuity of $g(\cdot, t)$; let $x_{n-1}$ satisfy

$$
\beta \leqslant x_{n-1} \leqslant \gamma \text {. }
$$

Then $x(t)$ exists, is unique on $t_{n-1} \leqslant t \leqslant t_{n}$, and satisfies the inequality (2.3) over this interval.

Proof. By our assumptions on $f$, all but one of the conditions of the Picard theorem are satisfied for equation (2.10). The missing condition (continuity of $g(x, t)$ as a function of $t)$ has been shown to be inessential. Thus an $x(t)$ exists which satisfies the integral equation

$$
x(t)=x_{n-1}+\int_{t_{u-1}}^{t} g\left[x\left(t^{\prime}\right), t^{\prime}\right] d t^{\prime}
$$

for all $t$ in $t_{n-1} \leqslant t \leqslant t_{n}$; furthermore, the points $(t, x(t))$ lie entirely within $\Delta S_{n}$, (2.17). Thus, in particular, $\alpha \leqslant x(t) \leqslant \delta$ for all $t \in\left[t_{n-1}, t_{n}\right]$.

The essential assertion of the lemma is (2.3), which is stronger (in general) than the condition that $[t, x(t)] \in \Delta S_{n}$.

Suppose there exists a $\tau, t_{n-1}<\tau \leqslant t_{n}$, such that $x(\tau)>\gamma$, thereby contradicting (2.3). $x\left(t_{n-1}\right)=x_{n-1} \leqslant \gamma$ by assumption (2.18). Since $x(t)$ satisfies (2.19), it is a continuous function of $t$. Hence there exists at least one value $\tau^{\prime}, t_{n-1} \leqslant \tau^{\prime}<\tau$, such that $x\left(r^{\prime}\right)=\gamma$.

Let $\boldsymbol{\tau}^{\prime}$ be the last such value; then we have:

$$
x\left(\tau^{\prime}\right)=\gamma \text { and } x(t)>\gamma \text { for } \tau^{\prime}<t \leqslant \tau .
$$

Furthermore $x(t) \leqslant \delta$ for all $t$ in $\left[t_{n-1}, t_{n}\right]$, by the Picard proof. Now substitute $t=\tau$ and $t=\tau^{\prime}$ into (2.19) and subtract. This gives:

$$
\begin{aligned}
x(\tau)-x\left(\tau^{\prime}\right) & =\int_{\tau}^{\tau^{\prime}} g[x(t), t] d t \\
& =\int_{\tau}^{\tau^{\prime}} f[x(t), v(t), t] d t .
\end{aligned}
$$

By our assumptions, $x(\tau)>\gamma$ and $x\left(\tau^{\prime}\right)=\gamma$ hence the left-hand side is positive. But by (2.20) and (2.2) the right-hand side is the integral of a negative function over a non-zero interval and is therefore negative. 
This contradiction destroys our assumption $x(\tau)>\gamma$, and thus we conclude $x(t) \leqslant \gamma$ for all $t_{n-1} \leqslant t \leqslant t_{n}$. A similar reductio ad absurdum establishes the inequality $x(t) \geqslant \beta$. This complete the proof.

THEOREM. Under the conditions stated, the integral equation (2.14) has a unique solution for all $t$ in $[0, T]$, and this solution $x(t)$ satisfies (2.3).

PrOOF. The proof is constructive; proceeding by constructing the solution $x(t)$ in successive intervals $t_{n-1} \leqslant t \leqslant t_{n}$, until we reach $t=T$.

At the first step, $n=1$, the initial value $x_{n-1}=x_{0}=x(0)=b$ satisfies (2.18), by (1.4). Hence we can construct $x(t)$ for $0 \leqslant t \leqslant t_{1}$ and the lemma ensures that $x_{1}=x\left(t_{1}\right)$ also satisfies (2.18).

We now reapply the lemma, with ever-increasing $n$, until we get to

$$
n=N=\text { integer part of }(T / \Delta t)+1 \text {. }
$$

The value $t=T$ lies within this final interval, and the lemma ensures that $x(t)$ exists up to $t=T$ and satisfies the inequality (2.3).

\section{Pulse variation of control}

In Section 2, we proved the existence, uniqueness and bounded nature of the solution $x(t)$ of the integral equation

$$
x(t)=b+\left.\right|_{0} ^{t} f\left[x\left(t^{\prime}\right), t\left(t^{\prime}\right), t^{\prime}\right] d t^{\prime}
$$

for all $0 \leqslant t \leqslant T$ and all admissible controls $v(t)$.

This does not prove the existence of an optimal control, $v^{*}(t)$, which satisfies

(ii) $v^{*}(t)$ is admissible,

(ii) for all admissible $v(t), x_{0}(T) \geqslant x_{0}^{*}(T)$, where $x_{0}(T)$ is defined by (1.1). Optimal controls may fail to exist in apparently quite ordinary conditions [2]. One can often exhibit sequences of admissible controls $v_{\alpha}(t)$, with uniformly decreasing cost $x_{0 \alpha}(T)$, for which the limit as $\alpha \rightarrow \infty$ either fails to exist as a function $v(t)$, or fails to be admissible. For instance, $v_{\alpha}(t)$ may be a piecewise continuous function with $\alpha$ points of discontinuity in $[0, T)$. Then the limiting function (if it exists) has infinitely many points of discontinuity, and is therefore no longer admissible.

In optimal control theory, the usual procedure is to look for necessary conditions. The existence of an optimal control $v^{*}(t)$ is assumed, and one deduces conditions which the function $v^{*}(t)$ must satisfy. 
Let $v^{*}(t)$ be an optimal control, and $x^{*}(t)$ be the corresponding trajectory which satisfies

$$
x^{*}(t)=b+\int_{0}^{t} f\left[x^{*}\left(t^{\prime}\right), v^{*}\left(t^{\prime}\right), t^{\prime}\right] d t^{\prime} .
$$

Following Pontryagin [8], we now consider a different control function $v(t)$, which equals $v^{*}(t)$ everywhere except within a short time interval $\tau-\varepsilon<t \leqslant \tau$, where

$$
\varepsilon>0, \quad 0<\tau \leqslant T, \quad 0 \leqslant \tau-\varepsilon<T .
$$

Furthermore, $\varepsilon$ is to be chosen small enough so that the interval $[\tau-\varepsilon, \tau)$ contains no discontinuity of $f(\cdot, \cdot, t)$ or $f_{0}(\cdot, \cdot, t)$ or $v^{*}(t)$. Since these functions have at most finite numbers of points of discontinuity, $\varepsilon$ can always be so chosen, even if $\tau$ is a point of discontinuity. Futhermore, $f(\cdot, \cdot, t), f_{0}(\cdot, \cdot, t)$ and $v^{*}(t)$ are left-hand continuous everywhere (by assumption) hence these functions are then continuous in the closed interval $[\tau-\varepsilon, \tau]$ as well.

The varied control is obtained by choosing any member $v \in \Omega$ of the control set, and setting

$$
v(t)= \begin{cases}v & \text { for } \tau-\varepsilon<t \leqslant \tau \\ v^{*}(t) & \text { for all other } t \text { in }[0, T]\end{cases}
$$

The work of Section 2 ensures that (3.1) has a solution $x(t)$ with this $v(t)$. We expect that $x(t)$ differs only little from $x^{*}(t)$ if $\varepsilon$ is sufficiently small. In the remainder of this section, we shall make this statement more precise.

Before doing so, however, let us make a qualitative observation concerning the essential difference between optimal control theory and the classical calculus of variations.

In the calculus of variations, the minimization of $x_{0}(T),(1.1)$, subject to (3.1) is quite a reasonable sort of problem. To tackle it, one starts by assuming that an optimal $v^{*}(t)$ exists, and one then considers a "varied control"

$$
v(t)=v^{*}(t)+\delta v(t)
$$

where $|\delta v(t)|$ is everywhere small and has other "nice" properties like being differentiable, being zero at $t=0$ and/or $t=T$, etc. The expansion is then essentially in powers of $\delta v(t)$.

In optimal control theory, however, we cannot guarantee that $\delta v(t)$ in (3 5) may be chosen arbitrarily small. To take an extreme but very practical example, assume the control set $\Omega$ is discrete with just two elements: $\Omega=\{0,1\}$. That is, the control is "on-off"; at any given time $t$, the control is either "on", $v(t)=1$, or "off", $v(t)=0$. There is nothing in between. (A common example is the thermostat control on a house-heating furnace [2].) 
With (3.5), it is apparent that $\delta v(t)$ can take exactly three values

$$
\delta v(t)=0,1 \text {, or }-1 \text {. }
$$

There is no way in which $\delta v(t)$ can be "uniformly small" unless $\delta v(t) \equiv 0$, all $t$, a most uninformative "variation of control".

Thus the basic approach of the calculus of variations appears to break down because $v(t)$ is restricted to values within some control set $\Omega$. (This apparent breakdown is less obvious, but stiii occurs, if $\Omega$ contains closed intervals $u \leqslant v \leqslant w)$. $\dagger$

To make any progress at all, it is necessary not only to have "varied controls", but varied controls containing a small parameter $\varepsilon>0$ of some sort, so that we can use expansions. In our view, the "pulse variation of control", equation (3.4), is the essential idea of modern control theory. The variation itself is not small; there is no reason to expect $\left|v-v^{*}(t)\right|$ to be small for any $t$ in $[\tau-\varepsilon, \tau]$, and nothing like that is assumed in (3.4). There is a small parameter $\varepsilon$, however: it is the time interval during which $v(t)$ differs from the optimal control $v^{*}(t)$.

The idea of a pulse variation, not the use of Lebesgue theory, is the essential contribution of Pontryagin [8]. Lebesgue theory, far from helping one's understanding, rather tends to obscure the elegant simplicity of the theory, drowning beautifully clear and powerful ideas in a mass of technicalities from the theory of functions of real variables.

After this digression, let us return to (3.1) and (3.2). Since $v(t)=v^{*}(t)$ for $0 \leqslant t \leqslant \tau-\varepsilon$, and since the solution is unique we conclude

$$
x(t)=x^{*}(t) \text { for } 0 \leqslant t \leqslant \tau-\varepsilon .
$$

We expect $x(t)$ to differ from $x^{*}(t)$ for $t>\tau-\varepsilon$, but not by much. Let us define:

$$
\xi(t) \equiv f\left[x^{*}(t), v, t\right]-f\left[x^{*}(t), v^{*}(t), t\right] \text { for } \tau-\varepsilon \leqslant t \leqslant \tau .
$$

Then (3.1), (3.2) and (3.6) imply

$$
\begin{aligned}
x(\tau)-x^{*}(\tau) & =\int_{\tau-8}^{\tau} \xi(t) d t+\int_{\tau-8}^{\tau}\left[f(x(t), v, t)-f\left(x^{*}(t), v, t\right)\right] d t \\
& =I_{1}+I_{2} .
\end{aligned}
$$

LEMMA. $I_{1}=\varepsilon \xi(\tau)+o(\varepsilon)$ and $I_{2}=O\left(\varepsilon^{2}\right)$ as $\varepsilon \rightarrow 0+$.

ProOF.

$$
\left(I_{1}-\varepsilon \xi(\tau)\right) / \varepsilon=\frac{1}{\varepsilon} \int_{\tau-8}^{\tau}[\xi(t)-\xi(\tau)] d t .
$$

† There are general theories of the first variation [4] containing both the calculus of variations and optimal control theory as particular instances. Such unifications involve conceptual and technical difficulties which make them unsuitable for an elementary treatment. 
The absolute value of this last expression is

$$
\leqslant \max _{\tau-\varepsilon \leqslant t \leqslant \tau}|\xi(t)-\xi(\tau)|
$$

which, because $\xi(t)$ is continuous for $\tau-\varepsilon \leqslant t \leqslant \tau$, tends to 0 as $\varepsilon \rightarrow 0+$. Thus the first estimate is verified. As for the second,

$$
\begin{aligned}
\left|I_{2}\right| & =\left|\int_{\tau-8}^{\tau} d t \int_{x^{*}(t)}^{x(t)} d x\left(\frac{\partial f}{\partial x}\right)\right| \\
& \leqslant\left|\int_{\tau-8}^{\tau} d t \int_{x^{*}(t)}^{x(t)} d x\right| \frac{\partial f}{\partial x}|| \\
& \leqslant\left|\int_{\tau-8}^{\tau} d t \int_{x^{*}(t)}^{x(t)} d x \cdot B\right| \\
& \leqslant B \int_{\tau-\varepsilon}^{\tau} d t\left|x(t)-x^{*}(t)\right| \leqslant B \int_{\tau-8}^{\tau} d t \cdot 2 M \varepsilon \\
& =2 M B \varepsilon^{2},
\end{aligned}
$$

where we have used (1.7a) in step 3 , and the second inequality of (2.17) in step 5 of the proof (with $t_{n-1}$ replaced by $\tau-\varepsilon$ ).

Intuitively, this lemma can be interpreted as follows: The difference $x(\tau)-x^{*}(\tau)$ is caused by a number of separate effects within the "active interval" $[\tau-\varepsilon, \tau]$; for example,

(1) $v$ differs from $v^{*}(\tau)$,

(2) $v^{*}(t)$ differs from $v^{*}(\tau)$,

(3) $x(t)$ as an argument of $f[x(t), v, t]$ differs from $x^{*}(t)$ in $f\left[x^{*}(t), v^{*}(t), t\right]$, and so on. According to our lemma, the first of the effects listed above is the dominant one for small $\varepsilon$, everything else is of higher order in $\varepsilon$. The net result is (this serves also as a definition of $\zeta(\tau)$ ):

$$
\begin{aligned}
x(\tau)-x^{*}(\tau) & =\varepsilon\left[f\left(x^{*}(\tau), v, \tau\right)-f\left(x^{*}(\tau), v^{*}(\tau), \tau\right)\right]+o(\varepsilon) \\
& \equiv \varepsilon \zeta(\tau)+o(\varepsilon) .
\end{aligned}
$$

Now let us establish a result for times $t \geqslant \tau$, in the interval $\tau \leqslant t \leqslant T$. Define $\chi(t)$ by

$$
\chi(t)=\frac{1}{\varepsilon}\left[x(t)-x^{*}(t)\right], \quad \tau \leqslant t \leqslant T .
$$

By subtracting the differential equations for $x$ and $x^{*}$ from each other, we get 
(except at a finite number of possible points of discontinuity)

$$
\left.\frac{d \chi}{d t}=\frac{1}{\varepsilon}\left\{f\left[x(t), v^{*}(t), t\right)\right]-f\left[x^{*}(t), v^{*}(t), t\right]\right\} .
$$

By assumption, $f(x, \cdot, \cdot)$ is twice differentiable with a bounded second derivative. We use Taylor's theorem with remainder term to get

$$
f\left(x, v^{*}, t\right)-f\left(x^{*}, v^{*}, t\right)=\left(\frac{\partial f}{\partial x}\right)^{*}\left(x-x^{*}\right)+\hat{R},
$$

where $(\partial f / \partial x)^{*}$ is evaluated at $\left(x^{*}(t), v^{*}(t), t\right)$ and the remainder $\hat{R}$ is bounded by using (1.6) and (3.10):

$$
|\hat{R}|=\left|\frac{1}{2}\left(\frac{\partial^{2} f}{\partial x^{2}}\right)_{x^{*}+\theta\left(x-x^{*}\right)}\left(x-x^{*}\right)^{2}\right| \leqslant \frac{1}{2} A \varepsilon^{2} \chi^{2}(t) .
$$

We therefore obtain from (3.11), (3.12), (3.13)

$$
\frac{d \chi}{d t}=\left(\frac{\partial f}{\partial x}\right)^{*} \chi+X(t)
$$

where

$$
|X(t)| \leqslant \frac{\varepsilon}{2} A \chi^{2}(t) .
$$

If we ignore the remainder term $X(t)$ for the moment, we get a linear differential equation. We shall call the dependent variable $\zeta(t)$. The equation is

$$
\frac{d \zeta}{d t}=\left(\frac{\partial f}{\partial x}\right)^{*} \zeta
$$

We note that $(\partial f / \partial x)^{*}$ is a known function of $t$, call it $F(t)$,

$$
\left.F(t) \equiv\left(\frac{\partial f}{\partial x}\right)^{*} \quad \text { (evaluated at } x^{*}(t), v^{*}(t), t\right) .
$$

But then (3.16) has an explicit solution in the interval under consideration (everywhere except at points of discontinuity of $F(t))$ :

$$
\zeta(t)=\zeta(\tau) \exp \left(\int_{\tau}^{t} F\left(t^{\prime}\right) d t^{\prime}\right) \text { for } \tau \leqslant t \leqslant T .
$$

We take as our initial condition the value $\zeta(\tau)$ defined by (3.9). We note that $\zeta(t)$ is bounded (see (3.17) and (1.7a)), and is zero only if $\zeta(\tau)=0$ (an uninteresting case).

With this preparation, we can state the

LeMmA. For $\tau \leqslant t \leqslant T$, it is true that

$$
x(t)=x^{*}(t)+\varepsilon \zeta(t)+o(\varepsilon)
$$

where $\zeta(t)$ is defined by (3.9), (3.17) and (3.18). 
Proof. Define $\chi(t)$ by (3.19) and put

$$
\chi(t)=\zeta(t) \rho(t) \quad \text { with } \rho(\tau)=1+o(1) .
$$

(This second equation above is a consequence of (3.10) and (3.9).) Substitute this into (3.14) to get

$$
\frac{d \rho}{d t}=\frac{1}{\zeta(t)} X(t)
$$

Use (3.15) and (3.20) to get

$$
\left|\frac{1}{\rho^{2}(t)} \frac{d \rho}{d t}\right| \leqslant \frac{\varepsilon}{2} A|\zeta(t)| .
$$

Since $\left(1 / \rho^{2}\right)(d \rho / d t)=-(d / d t)(1 / \rho)$, we obtain the bound:

$$
\begin{aligned}
\left|\frac{1}{\rho(\tau)}-\frac{1}{\rho(t)}\right| & =\left|\int_{\tau}^{t} \frac{1}{\rho^{2}} \frac{d \rho}{d t^{\prime}} d t^{\prime}\right| \\
& \leqslant \frac{\varepsilon}{2} A \int_{\tau}^{t}\left|\zeta\left(t^{\prime}\right)\right| d t^{\prime} \leqslant \frac{\varepsilon}{2} A \int_{\tau}^{T}\left|\zeta\left(t^{\prime}\right)\right| d t^{\prime}=A^{\prime} \varepsilon .
\end{aligned}
$$

This bound together with (3.20) means that, for sufficiently small $\varepsilon$,

$$
\rho(t)=1+o(1), \quad \tau \leqslant t \leqslant T,
$$

and thus (3.19) follows from (3.29) and (3.10). This completes the lemma.

To sum up: the essential step is the introduction of the varied control $v(t),(3.4)$. This control results in a state function $x(t)$ which is related to the optimal $x^{*}(t)$ by (3.19), with

$$
\begin{aligned}
\zeta(t) & =0 \text { for } 0 \leqslant t \leqslant \tau-\varepsilon, \\
\zeta(\tau) & =f\left[x^{*}(\tau), v, \tau\right]-f\left[x^{*}(\tau), v^{*}(\tau), \tau\right], \\
\frac{d \zeta}{d t} & =\left(\frac{\partial f}{\partial x}\right)^{*} \zeta \text { for } \tau<t \leqslant T .
\end{aligned}
$$

In this form, the result generalizes well. The explicit solution (3.18) of (3.22c) does not generalize to control problems in which $\mathbf{x}(t)$ is a vector variable with $n \geqslant 2$ components; but an alternative proof of (3.19), based on the Gronwall-Bellman inequality, can be given for that case.

For later work, we note that an entirely similar argument goes through for $x_{0}(t)$, defined by

$$
x_{0}(t)=\int_{0}^{t} f_{0}\left[x\left(t^{\prime}\right), v\left(t^{\prime}\right), t^{\prime}\right] d t^{\prime}
$$


We note that, for $t=T, x_{0}(T)$ is the quantity to be minimized in (1.1). For $0 \leqslant t \leqslant T$, at all but at most a finite number of points, $x_{0}(t)$ satisfies the differential equation

$$
\frac{d x_{0}}{d t}=f_{0}[x(t), v(t), t]
$$

with the initial condition

$$
x_{0}(t)=0 \text {. }
$$

No existence proof is necessary here: (3.23) is the explicit solution, known as soon as $v(t)$ and $x(t)$ are known. Let $x_{0}^{*}(t)$ be the function

$$
x_{0}^{*}(t)=\int_{0}^{t} f_{0}\left[x^{*}\left(t^{\prime}\right), v^{*}\left(t^{\prime}\right), t^{\prime}\right] d t^{\prime}
$$

obtained from the optimal control $v^{*}(t)$, and let $x(t)$ in (3.23) be obtained from the varied control (3.4).

It is then easy to show, by arguments completely similar to the ones before, that

$$
\begin{aligned}
x_{0}(t) & =x_{0}^{*}(t)+\varepsilon \zeta_{0}(t)+o(\varepsilon), \quad 0 \leqslant t \leqslant T, \\
\zeta_{0}(t) & =0 \quad \text { for } 0 \leqslant t \leqslant \tau-\varepsilon, \\
\zeta_{0}(\tau) & =f_{0}\left[x^{*}(\tau), v, \tau\right]-f_{0}\left[x^{*}(\tau), v^{*}(\tau), \tau\right], \\
\frac{d \zeta_{0}}{d t} & =\left(\frac{\partial f_{0}}{\partial x}\right)^{*} \zeta \quad \text { for } \tau<t \leqslant T .
\end{aligned}
$$

There is one essential thing to notice about (3.28c): the right-hand side of $(3.28 \mathrm{c})$ is proportional to $\zeta(t)$, not to $\zeta_{0}(t)$. This arises from (3.23)-the right-hand side of (3.23) depends on $x(t)$, not on $x_{0}(t)$. Except for this, (3.28) is very similar to (3.22).

\section{The maximum principle}

Following Pontryagin [8] we introduce the Hamiltonian $\dagger$

$$
H=H(\lambda, x, v, t)=\lambda f(x, v, t)-f_{0}(x, v, t)
$$

† Pontryagin defines the Hamiltonian by $H=\lambda f+\lambda_{0} f_{0}$, with $t w o$ co-state variables $\lambda$ and $\lambda_{0}$. Subsequently $\lambda_{0}$ turns out to be constant in time; its value is determined in the Pontryagin theory as follows:

$\lambda_{0}=-1$ if $v(t)$ is feasible and $x_{0}(T),(1.1)$, is to be minimized;

$\lambda_{0}=+1$ if $v(t)$ is feasible and $x_{0}(T),(1.1)$, is to be maximized;

$\lambda_{0}=0$ if $v(t)$ is unfeasible.

For our simplified problem with its free end-point, Section 2 showed that all admissible controls are feasible, and we have agreed to look for a minimum of $x_{0}(T)$. Thus $\lambda_{0}=-1$, and there is no need to introduce $\lambda_{0}$ at all.

The signs in (4.1) are chosen so as to obtain a maximum principle in the final inequality (4.12). If one thinks of $\lambda(t)$ as a Lagrange multiplier, a more conventional form should be $H^{\prime}=f_{0}-\lambda f$, but this reverses the inequality in (4.12) and thus gives a minimum principle. We follow Pontryagin's choice of signs. 
and we observe that the differential equation (1.2) is then the first of the two canonical equations

$$
\frac{d x}{d t}=\frac{\partial H}{\partial \lambda}, \frac{d \lambda}{d t}=-\frac{\partial H}{\partial x}
$$

The second of these equations determines the time development of the "co-state variable" $\lambda(t): \dagger$

$$
\frac{d \lambda}{d t}=\frac{\partial f_{0}}{\partial x}-\lambda \frac{\partial f}{\partial x}
$$

We now define:

$$
Z(t)=\lambda(t) \zeta(t)-\zeta_{0}(t)
$$

where $\lambda(t)$ satisfies (4.3), $\zeta(t)$ is given by (3.22) and $\zeta_{0}(t)$ by (3.28).

Except at a finite number of isolated points of discontinuity in $t$, the differential equations (3.22c), (3.28c) and (4.3) are satisfied for all $\tau \leqslant t \leqslant T$. In that region, straightforward differentiation gives

$$
\frac{d Z}{d t}=0 \quad \text { for } \tau \leqslant t \leqslant T
$$

so that, in particular,

$$
Z(\tau)=Z(T)
$$

(the functions entering (4.4) are continuous, so (4.6) follows even if (4.5) fails to hold at isolated points).

By assumption, $v^{*}(t)$ is an optimal control, and any varied control, such as $v(t)$, gives a result which is no better than $x_{0}^{*}(T)$ :

$$
x_{0}(T) \geqslant x_{0}^{*}(T) \text { for all variations of control. }
$$

Now put $t=T$ in (3.27) and assume $\varepsilon$ is small enough so that terms of $o(\varepsilon)$ can be ignored. We get from (4.7)

$$
\zeta_{0}(T)=\lim _{\varepsilon \rightarrow 0+} \frac{x_{0}(T)-x_{0}^{*}(T)}{\varepsilon} \geqslant 0 .
$$

We can relate this result to $Z(T)$ if we impose the following end-point condition on the function $\lambda(t)$ :

$$
\lambda(T)=0
$$

for then

$$
Z(T)=\lambda(T) \zeta(T)-\zeta_{0}(T)=-\zeta_{0}(T) \leqslant 0
$$

$\dagger$ For a geometric interpretation of the co-state variable see Halkin [6]. 
But by (4.6) it follows that $Z(\tau) \leqslant 0$, also. Hence we have, using (3.22b) and (3.28b), and then (4.1):

$$
\begin{aligned}
Z(\tau) & =\lambda(\tau) \zeta(\tau)-\zeta_{0}(\tau) \\
& =\lambda(\tau)\left[f\left(x^{*}(\tau), v, \tau\right)-f\left(x^{*}(\tau), v^{*}(\tau), \tau\right)\right]-\left[f_{0}\left(x^{*}(\tau), v, \tau\right)-f_{0}\left(x^{*}(\tau), v^{*}(\tau), \tau\right)\right] \\
& =H\left[\lambda(\tau), x^{*}(\tau), v, \tau\right]-H\left[\lambda(\tau), x^{*}(\tau), v^{*}(\tau), \tau\right] \leqslant 0 .
\end{aligned}
$$

The finai inequality is equivalent to the Pontryagin maximum principle:

$$
H\left[\lambda(\tau), x^{*}(\tau), v^{*}(\tau), \tau\right] \geqslant H\left[\lambda(\tau), x^{*}(\tau), v, \tau\right]
$$

the inequality holding for all $\tau$ in $0<\tau \leqslant T$ and for all $v \in \Omega$, the control set. The inequality asserts that the optimal control $v^{*}(\tau)$ at any time $\tau$ is such as to maximize the Hamiltonian.

This concludes our elementary derivation of the Pontryagin maximum principle.

\section{REFERENCES}

[1] R. P. Agnew, Differential equations (McGraw-Hill, New York and London, 1942). (See in particular Chapter 15.)

[2] J. M. Blatt, "Heating your house with control theory" (unpublished).

[3] G. F. Bryant and D. O. Mayne, "The maximum principle", Int. J. Control, 20 (1974), 1021-1054.

[4] R. V. Gamkrelidze, "Extremal problems in finite dimensional spaces", J. Optimization Th. Appl. 1 (1967), 173-193; and L. D. Berkovitz, "Variational methods in problems of control and programming", J. Math. Anal. Applic. 3 (1961), 145-169; and D. F. Lawden, Optimal trajectories for space navigation (Butterworths, London, 1963), Section 1.9.

[5] G. Hadley and M. C. Kemp, Variational methods in economics (American Elsevier Publishing Co., Inc., New York; North-Holland Publishing Co., Amsterdam-London, 1971).

[6] H. Halkin in C. Leitmann (ed.), Topics in optimization (Academic Press, New York and London, 1967), pp. 197-262.

[7] H. Halkin and L. W. Neustadt, "General necessary conditions for optimization problems", Proc. Nat. Acad. Sci. U.S.A. 56 (1966), 1066-1071.

[8] L. S. Pontryagin, V. G. Boltyanskii, R. V. Gramkrelidze and E. F. Mischenko, The mathematical theory of optimal processes (Interscience Publishers, Inc., New York, 1962).

School of Mathematics

University of New South Wales

Kensington, N.S.W. 2033, Australia 